\title{
Time-Resolved Measurement of a Charge Qubit
}

\author{
Georg M. Reuther, David Zueco, Peter Hänggi, and Sigmund Kohler \\ Institut für Physik, Universität Augsburg, Universitätsstraße 1, D-86135 Augsburg, Germany
}

(Dated: November 29, 2018)

\begin{abstract}
We propose a scheme for monitoring coherent quantum dynamics with good time-resolution and low backaction, which relies on the response of the considered quantum system to high-frequency ac driving. An approximate analytical solution of the corresponding quantum master equation reveals that the phase of an outgoing signal, which can directly be measured in an experiment with lock-in technique, is proportional to the expectation value of a particular system observable. This result is corroborated by the numerical solution of the master equation for a charge qubit realized with a Cooper-pair box, where we focus on monitoring coherent oscillations.
\end{abstract}

PACS numbers: 42.50.Dv, 03.65.Yz, 03.67.Lx, 85.25.Cp

An indispensable requirement for a quantum computer is the readout of its state after performing gate operations. For that purpose it is sufficient to distinguish between two possible logical states. At the same time, it is desirable to demonstrate the coherence of time evolution explicitly. For solid-state qubits, this has been accomplished by Rabi-type experiments [1, 2]. In general the qubit state measurement is destructive, so that an interference pattern emerges only after a number of experimental runs. Certainly, it would be preferable to observe signatures of coherent dynamics already in a single run.

Both the charge and the flux degree of freedom of superconducting qubits can be measured by coupling the qubit to a low-frequency "tank" circuit that is excited resonantly [3, 4]. In doing so one makes use of the fact that the resonance frequency of the slow oscillator depends on the qubit state which, in turn, influences the phase of the oscillator response [3, 4, 5]. The drawback of this scheme, however, is that the coherent qubit dynamics is considerably faster than the driving. Thus, one can only observe the time-average of the qubit state, but not time-resolve its dynamics. Measuring the qubit by driving it at resonance is possible as well [6]. This however induces Rabi oscillations, making the qubit dynamics differ significantly from the undriven case [7, [8].

Here, by contrast, we propose to probe the qubit by a weak high-frequency driving that directly acts upon the qubit without a tank circuit being present. We find that the resulting outgoing signal possesses sidebands which are related to a phase shift and demonstrate that the latter is related to a qubit observable. Validating this relation numerically for a Cooper-pair box, we show that the underlying measurement scheme principally enables monitoring the coherent qubit dynamics experimentally in a single run with good time-resolution and fidelity, whereas the backaction on the qubit, induced by the driving, stays at a tolerable level.

Dissipative quantum circuit. - Although later on we focus on the dynamics of a superconducting charge qubit as sketched in Fig. 1] our measurement scheme is rather generic and can be applied to any open quantum system.
We employ the system-bath Hamiltonian [9, 10, 11]

$$
H=H_{0}+\sum_{k}\left(\frac{p_{k}^{2}}{2 L_{k}}+\frac{\left(q_{k}-\lambda_{k} C_{k} Q\right)^{2}}{2 C_{k}}\right),
$$

where $H_{0}$ denotes the system Hamiltonian and $Q$ is a system operator. The bath is modelled by $L C$ circuits with charges $q_{k}$ and conjugate momenta $p_{k}$, where $C_{k}$ and $L_{k}$ are effective capacitances and inductances, respectively, and $\lambda_{k}$ are the corresponding coupling constants. It is convenient to introduce the spectral density $I(\omega)=\frac{\pi}{2} \sum_{k} \lambda_{k}^{2}\left(C_{k} / L_{k}\right)^{1 / 2} \delta\left(\omega-\omega_{k}\right)$ which we assume to be ohmic, i.e. $I(\omega)=\omega Z_{0}$ with an effective impedance $Z_{0}$ [11, 12, 13]. By standard techniques we obtain the Bloch-Redfield master equation for the reduced system density operator $\rho$ in the weak-coupling limit [14],

$$
\dot{\rho}=-\frac{i}{\hbar}\left[H_{0}, \rho\right]-\frac{1}{\hbar}[Q,[\hat{Q}, \rho]]-i \frac{Z_{0}}{\hbar}\left[Q,[\dot{Q}, \rho]_{+}\right],
$$

with the anticommutator $[A, B]_{+}=A B+B A$ and the operators $\dot{Q}=i\left[H_{0}, Q\right] / \hbar$ and

$$
\hat{Q}=\frac{1}{\pi} \int_{0}^{\infty} d \tau \int_{0}^{\infty} d \omega S(\omega) \cos (\omega \tau) \tilde{Q}(-\tau)
$$

Here, $S(\omega)=I(\omega) \operatorname{coth}\left(\hbar \omega / 2 k_{\mathrm{B}} T\right)$ is the Fourier transform of the symmetrically ordered equilibrium correlation function $\frac{1}{2}\left\langle\left[\xi_{\text {in }}(\tau), \xi_{\text {in }}(0)\right]_{+}\right\rangle_{\text {eq }}$ at temperature $T$ with regard to the collective bath coordinate $\xi_{\text {in }}=\sum_{k} \lambda_{k} q_{k}$. The notation $\tilde{X}(t)$ is a shorthand for the Heisenberg operator $U_{0}^{\dagger}(t) X U_{0}(t)$, where $U_{0}$ is the system propagator.

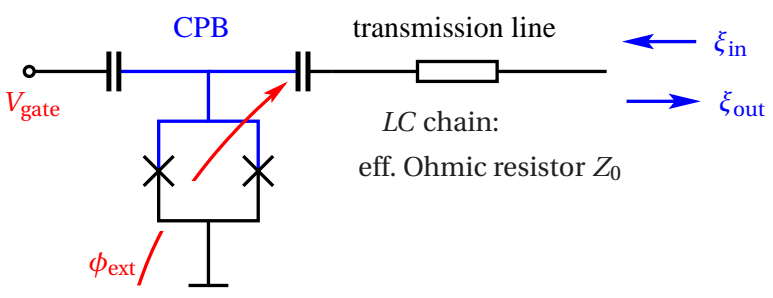

FIG. 1: (color online) Cooper pair box (CPB) coupled to a transmission line with ohmic effective impedance $Z_{0}$. 
In order to relate the quantum dynamics of the central circuit to the response via the transmission line, we employ the input-output formalism [15] which is an established tool in quantum optics and has also been used in quantum circuit theory [5]. It starts from the Heisenberg equation of motion for the environmental mode $k$ which reads $\ddot{q}_{k}+\omega_{k}^{2} q_{k}=\omega_{k}^{2} Q$, where $\omega_{k}=\left(L_{k} C_{k}\right)^{-1 / 2}$ denotes the angular frequency of mode $k$. Owing to its linearity, this equation of motion can be solved formally. Inserting the obtained solution into the Heisenberg equations of motion for the system operators, one arrives at the so-called quantum Langevin equation [16, 17]. For an ohmic environment, the latter possesses the inhomogeneity $\xi_{\text {in }}(t)-Z_{0} \dot{Q}(t)$, where the noise operator $\xi_{\text {in }}$ is fully determined by the correlation functions given above.

Alternatively, one can write the quantum Langevin equation in terms of the outgoing fluctuations [15]. The result differs only by the sign of the dissipative term, so that the inhomogeneity now reads $\xi_{\text {out }}(t)+Z_{0} \dot{Q}(t)$. The difference between these two equations relates the input and the output fluctuations via

$$
\xi_{\text {out }}-\xi_{\text {in }}=-2 Z_{0} \dot{Q}=-\frac{2 i Z_{0}}{\hbar}\left[H_{0}, Q\right],
$$

which is a cornerstone of the input-output formalism [15] and holds for any weakly coupled $\xi_{\text {in }}$. We used that for weak dissipation, $\dot{Q} \approx i\left[H_{0}, Q\right] / \hbar$ is essentially bathindependent.

Response to high-frequency driving.-We next probe the system by driving it via the transmission line with an ac signal $A \cos (\Omega t)$ that also couples to the system operator $Q$. Then, the Hamiltonian acquires an additional term: $H_{0} \rightarrow H_{0}+Q A \cos (\Omega t)$, and the master equation (2) changes accordingly. For the input $\xi_{\text {in }}$, this corresponds to one coherently excited incoming mode such that $\left\langle\xi_{\text {in }}(t)\right\rangle=A \cos (\Omega t)$, while the r.h.s. of the inputoutput relation (4) remains unchanged.

Because the driving must not significantly alter the system dynamics, we assume that the amplitude $A$ is sufficiently small, so that the driving can be treated perturbatively. This yields the ansatz $\rho(t)=\rho_{0}(t)+\rho_{1}(t)$, where $\rho_{0}(t)$ is the unperturbed state. To lowest order in $A, \rho_{1}$ obeys $\dot{\rho}_{1}=\mathcal{L}_{0} \rho_{1}-\frac{i}{\hbar} A\left[Q, \rho_{0}\right] \cos (\Omega t)$, where $\mathcal{L}_{0}$ denotes the superoperator on the r.h.s. of Eq. (2). This linear inhomogeneous equation of motion can be solved formally in terms of a convolution between the propagator of the undriven system and the inhomogeneity. If the driving frequency $\Omega$ is much larger than all relevant system frequencies, one may separate time scales to obtain

$$
\rho(t)=\rho_{0}(t)-i \frac{A}{\hbar \Omega}\left[Q, \rho_{0}(t)\right] \sin (\Omega t),
$$

which identifies $e A / \hbar \Omega$ as the necessarily small perturbation parameter. Together with the input-output relation (4) this solution allows us to compute the response of the system.
In an experiment it is possible to employ a lock-in technique with the incoming signal providing the reference oscillator. This singles out the high-frequency components of the outgoing signal, which correspond to the second term of the density operator (5) and read

$$
\left\langle\xi_{\text {out }}(t)\right\rangle=A \cos (\Omega t)+\frac{2 A Z_{0}}{\hbar^{2} \Omega}\left\langle\left[\left[H_{0}, Q\right], Q\right]\right\rangle_{0} \sin (\Omega t),
$$

where $\langle\ldots\rangle_{0}=\operatorname{tr}\left[\rho_{0}(t) \ldots\right]$ refers to the undriven dynamics. Writing next $\left\langle\xi_{\text {out }}(t)\right\rangle=A^{\prime} \cos \left[\Omega t-\phi_{\mathrm{hf}}^{0}(t)\right]$, we find the central expression

$$
\phi_{\mathrm{hf}}^{0}(t)=\frac{2 Z_{0}}{\hbar^{2} \Omega}\left\langle\left[\left[H_{0}, Q\right], Q\right]\right\rangle_{0},
$$

which relates a small phase shift $\phi_{\mathrm{hf}}^{0}(t)$ between the input and the output signal to a hermitian system observable describing the unperturbed low-frequency system dynamics. This means that the time-resolved evolution of the open quantum system can be monitored in a single run by continuously measuring the phase shift $\phi_{\mathrm{hf}}^{0}(t)$ with appropriate experimental techniques. In this connection, Eq. (7) represents the basis for our proposed measurement scheme. Below we will explore its validity and limitations for a specific system by comparing the phase of the output $\left\langle\xi_{\text {out }}(t)\right\rangle$ with the expectation value $\left\langle\left[\left[H_{0}, Q\right], Q\right]\right\rangle_{0}$, both computed from the numerical solution of the master equation (2) in the presence of ac driving.

Monitoring coherent qubit dynamics.-A particular case of a quantum circuit which recently attracted much interest is a Cooper pair box (CPB) which is sketched in Fig. 1 and described by the Hamiltonian

$$
H_{0}^{\mathrm{CPB}}=4 E_{\mathrm{C}}\left(\hat{N}-N_{g}\right)^{2}-\frac{E_{\mathrm{J}}}{2} \sum_{N=-\infty}^{\infty}(|N+1\rangle\langle N|+\text { h.c. }),
$$

where $N$ is the number of excess Cooper pairs in the box, so that the charge operator reads $Q=2 e \hat{N}=$ $2 e \sum_{N} N|N\rangle\langle N|$. The charging energy $E_{\mathrm{C}}$ is determined by various capacitances, while the scaled gate voltage $N_{g}$ and the effective Josephson energy $E_{\mathrm{J}}$ are controllable. If the charging energy is sufficiently large and $N_{g} \approx 1 / 2$, only the two charge states $|0\rangle \equiv|\downarrow\rangle$ and $|1\rangle \equiv|\uparrow\rangle$ matter and form a qubit [1, 4, 11] described by the Hamiltonian

$$
H_{0}^{\mathrm{qb}}=-\frac{1}{2} E_{\mathrm{el}} \sigma_{z}-\frac{1}{2} E_{\mathrm{J}} \sigma_{x},
$$

where the Pauli matrices $\sigma_{i}$ are defined in the qubit subspace and $E_{\mathrm{el}}=4 E_{\mathrm{C}}\left(1-2 N_{g}\right)$. The qubit energy splitting reads $\hbar \omega_{\mathrm{qb}}=\left(E_{\mathrm{el}}^{2}+E_{\mathrm{J}}^{2}\right)^{1 / 2}$. Moreover, $Q_{\mathrm{qb}}=e \sigma_{z}$ while by virtue of relation (77) the phase of the output signal is linked to the qubit observable $\sigma_{x}$ according to

$$
\phi_{\mathrm{hf}}^{0}(t)=-\frac{4 e^{2} Z_{0} E_{\mathrm{J}}}{\hbar^{2} \Omega}\left\langle\sigma_{x}(t)\right\rangle_{0} .
$$



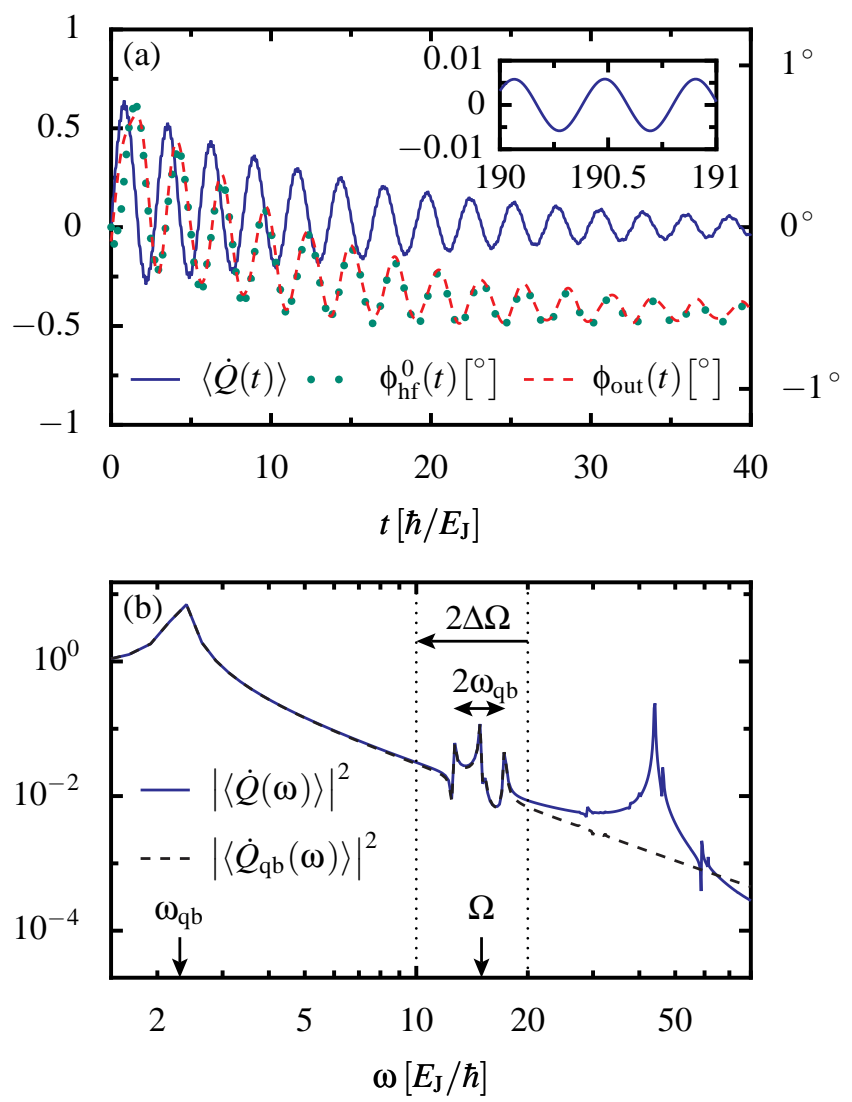

FIG. 2: (color online) Decaying qubit oscillations with initial state $|\uparrow\rangle$ in a weakly probed $\mathrm{CPB}$ with 6 states for $\alpha=Z_{0} e^{2} / \hbar=0.08, A=0.1 E_{\mathrm{J}} / e, E_{\mathrm{C}}=5.25 E_{\mathrm{J}}$ and $N_{g}=0.45$, so that $E_{\mathrm{el}}=2.1 E_{\mathrm{J}}$ and $\omega_{\mathrm{qb}}=2.3 E_{\mathrm{J}} / \hbar$. (a) Time evolution of the measured difference signal $\langle\dot{Q}\rangle \propto\left\langle\xi_{\text {out }}\right\rangle-\left\langle\xi_{\text {in }}\right\rangle$ (in units of $2 e E_{\mathrm{J}} / \hbar$ ) of the full $\mathrm{CPB}$ and its lock-in amplified phase $\phi_{\text {out }}$ (frequency window $\Delta \Omega=5 E_{\mathrm{J}} / \hbar$ ), compared to the estimated phase $\phi_{\mathrm{hf}}^{0} \propto\left\langle\sigma_{x}\right\rangle_{0}$ in the qubit approximation. The inset resolves the underlying small rapid oscillations with frequency $\Omega=15 E_{\mathrm{J}} / \hbar$ in the long-time limit. (b) Power spectrum of $\langle\dot{Q}\rangle$ for the full CPB Hamiltonian (solid) and for the two-level approximation (dashed).

This means that the high-frequency component of $\langle\dot{Q}\rangle$, which is manifest in the phase of the outgoing signal (4), contains information about the low-frequency qubit dynamics in terms of the unperturbed $\left\langle\sigma_{x}\right\rangle_{0}$.

We now turn to the question how relation (10) allows one to retrieve information about the coherent qubit dynamics in an experiment. Figure 2 (a) shows the time evolution of the expectation value $\langle\dot{Q}(t)\rangle$ for the initial state $|\uparrow\rangle \equiv|1\rangle$, obtained via numerical integration of the master equation (2) with the full Cooper-pair box Hamiltonian (8) in the presence of the ac driving which in principle may excite higher states. The driving, due to its rather small amplitude, is barely noticeable on the scale chosen for the main figure, but only on a refined scale for long times; see inset of Fig. 2(a). This already insinuates that the backaction on the dynamics is weak. In the cor- responding power spectrum of $\langle\dot{Q}\rangle$ depicted in Fig. 2(b), the driving is nevertheless reflected in sideband peaks at the frequencies $\Omega$ and $\Omega \pm \omega_{\mathrm{qb}}$. In the time domain these peaks correspond to a signal $\cos \left[\Omega t-\phi_{\text {out }}(t)\right]$. Moreover, non-qubit CPB states leads to additional peaks at higher frequencies, while their influence at frequencies $\omega \lesssim \Omega$ is minor. Experimentally, the phase $\phi_{\text {out }}(t)$ can be retrieved by lock-in amplification of the output signal, which we mimic numerically in the following way [18]: We only consider the spectrum of $\xi_{\text {out }}$ in a window $\Omega \pm \Delta \Omega$ around the driving frequency and shift it by $-\Omega$. The inverse Fourier transformation to the time domain provides $\phi_{\text {out }}(t)$ which is expected to agree with $\phi_{\mathrm{hf}}^{0}(t)$ and, according to Eq. (10), to reflect the unperturbed time evolution of $\left\langle\sigma_{x}\right\rangle_{0}$ with respect to the qubit. Although the condition of high-frequency probing, $\Omega \gg \omega_{\mathrm{qb}}$, is not strictly fulfilled and despite the presence of higher charge states, the lock-in amplified phase $\phi_{\text {out }}(t)$ and the predicted phase $\phi_{\mathrm{hf}}^{0}(t)$ are barely distinguishable for an appropriate choice of parameters as is shown in Fig. 2(a).

In order to quantify this agreement, we introduce the measurement fidelity $F=\left(\phi_{\text {out }},\left\langle\sigma_{x}\right\rangle_{0}\right)$, where $(f, g)=$ $\int d t f g /\left(\int d t f^{2} \int d t g^{2}\right)^{1 / 2}$ with time integration over the decay duration. Thus, the ideal value $F=1$ is assumed if $\phi_{\text {out }}(t)$ and $\left\langle\sigma_{x}(t)\right\rangle_{0}$ are proportional to each other, i.e. if the agreement between the measured phase and the unperturbed expectation value $\left\langle\sigma_{x}\right\rangle_{0}$ is perfect. Figure 3(a) depicts the fidelity as a function of the driving frequency. As expected, whenever non-qubit CPB states are excited resonantly, we find $F \ll 1$, indicating a significant population of these states. Far-off such resonances, the fidelity increases with the driving frequency $\Omega$. A proper frequency lies in the middle between the qubit doublet and the next higher state. In the present case, $\Omega \approx 15 E_{\mathrm{J}} / \hbar$ appears as a good choice. Concerning the driving amplitude, one has to find a compromise, because as $A$ increases, so does the phase contrast of the outgoing signal (6), while the driving perturbs more and more the low-frequency dynamics. For the frequency chosen above, the fidelity is best in the range $A=0.1-1 \hbar \omega_{\mathrm{qb}} / e$. This corresponds to $e A / \hbar \Omega \approx 10^{-2}-10^{-3}$, which justifies our perturbative treatment.

Measurement quality and backaction.-For any quantum measurement, one has to worry about backaction on the system in terms of decoherence. In our measurement scheme, decoherence plays a particular role, because both the driving and the ohmic environment couple to the $\mathrm{CPB}$ via the same mechanism. This is reflected by the fact that the predicted phase (10) is proportional to the dimensionless dissipation strength $\alpha=Z_{0} e^{2} / \hbar$. However, $\alpha$ should not exceed 0.1 in order to preserve a predominantly coherent time evolution, which also means that our measurement is weak, but destructive. The condition $\alpha \lesssim 0.1$ together with the above conditions on the driving amplitude and frequency provides phase shifts $\phi_{\text {out }}$ of the order $1^{\circ}$, which is small but still measurable 


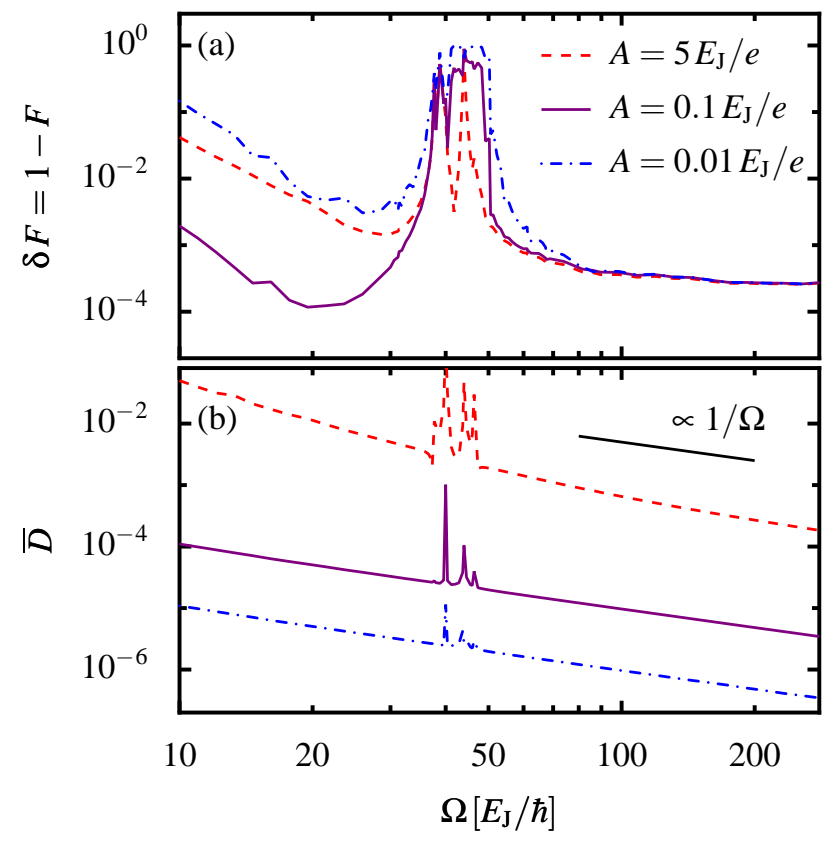

FIG. 3: (color online) (a) Fidelity defect $\delta F=1-F$ and (b) time-averaged trace distance between the driven and the undriven density operator of the CPB for various driving amplitudes as a function of the driving frequency. All other parameters are the same as in Fig. 2.

with present technologies. The additional decoherence due to the driving, by contrast, is not noticeable. This is in agreement with the first-order result $\operatorname{tr}\left(\rho^{2}\right)=\operatorname{tr}\left(\rho_{0}^{2}\right)$ which follows from Eq. (5).

In order to investigate to what extent the driving affects the quantum state of the $\mathrm{CPB}$, we compute the trace distance $D(t)=\frac{1}{2} \operatorname{tr}\left|\rho(t)-\rho_{0}(t)\right|$ [19] between the density operators of the driven system $\rho(t)$ and the undriven reference $\rho_{0}(t)$. Its time average $\bar{D}$ quantifies the perturbation due to the driving. Figure 3 (b) indicates that $\bar{D} \propto A / \Omega$, unless the driving is in resonance with higher levels. This confirms the picture drawn by studying the measurement fidelity $F$. For practically all parameters used in Fig. 3, we found that the total population of levels outside the qubit doublet is always less than $0.1 \%$. The only exception occurs again in the case of resonances with non-qubit CPB levels. Far from these resonances, the system is faithfully described with only the qubit levels.

In our investigations, we have not considered excitations of quasiparticles which are relevant once the driving frequency becomes of the order of the gap frequency of the superconducting material. Thus, for an aluminum CPB our model is valid only for $\Omega \lesssim 100 \mathrm{GHz}$. Since a typical Josephson energy is of the order of some $\mathrm{GHz}$, a driving frequency $\Omega \approx 20 E_{\mathrm{J}} / \hbar$ is still within this range while it provides already a good measurement quality.

Conclusions. - We have proposed a method for the time-resolved monitoring of the dynamics of a quantum system coupled to a dissipative environment. The crucial requirement for this is the possibility to drive the system coherently via one environmental high-frequency mode accompanied by measuring the phase of the outgoing signal via lock-in techniques. By analyzing the highfrequency response, we have found that the phase of the output signal is related to a particular system observable. We have substantiated this relation by computing both quantities numerically for a charge qubit implemented with a Cooper-pair box. For decaying coherent oscillations, we have demonstrated experimental feasibility on condition that the coupling to the environment is not too weak and that the driving frequency exceeds the qubit splitting and is off resonance with higher levels. Then the measurement fidelity is rather good, while the lowfrequency qubit dynamics is almost not affected by the driving and transitions to higher levels do not play a relevant role. The implementation of our scheme will enable the demonstration of quantum coherence of solid-state qubits in single-shot experiments.

This work has been supported by the DFG through SFB 631 and by the German Excellence Initiative via "Nanosystems Initiative Munich (NIM)".

[1] D. Vion et al., Science 296, 886 (2002).

[2] I. Chiorescu, Y. Nakamura, C. J. P. Harmans, and J. E. Mooij, Science 299, 1869 (2003).

[3] M. A. Sillanpää et al., Phys. Rev. Lett. 95, 206806 (2005).

[4] M. Grajcar et al., Phys. Rev. B 69, 060501(R) (2004).

[5] G. Johansson, L. Tornberg, and C. M. Wilson, Phys. Rev. B 74, 100504(R) (2006).

[6] Ya. S. Greenberg, E. Il'ichev, and A. Izmalkov, Europhys. Lett. 72, 880 (2005).

[7] Y.-X. Liu, C. P. Sun, and F. Nori, Phys. Rev. A 74, 052321 (2006).

[8] C. M. Wilson et al., Phys. Rev. Lett. 98, 257003 (2007).

[9] A. J. Leggett et al., Rev. Mod. Phys. 59, 1 (1987).

[10] P. Hänggi, P. Talkner, and M. Borkovec, Rev. Mod. Phys. 62, 251 (1990).

[11] Y. Makhlin, G. Schön, and A. Shnirman, Rev. Mod. Phys. 73, 357 (2001).

[12] B. Yurke and J. S. Denker, Phys. Rev. A 29, 1419 (1984).

[13] M. H. Devoret, in Quantum Fluctuations in Electrical circuits, Les Houches, Session LXIII (Elsevier, Amsterdam, 1995), Chap. 10.

[14] K. Blum, Density Matrix Theory and Applications (Springer, New York, 1996).

[15] C. W. Gardiner and M. J. Collett, Phys. Rev. A 31, 3761 (1985).

[16] G. W. Ford, J. T. Lewis, and R. F. O'Connell, J. Stat. Phys. 53, 439 (1988).

[17] P. Hänggi and G.-L. Ingold, Chaos 15, 026105 (2005).

[18] J. H. Scofield, Am. J. Phys. 62, 129 (1994).

[19] M. A. Nielsen and I. L. Chuang, Quantum Computing and Quantum Information (Cambridge University Press, Cambridge, 2000). 